\title{
An Improved Iterative Nonlinear Least Square Approximation Method for the Design of SISO Wideband Mobile Radio Channel Simulators
}

\author{
Akmal Fayziyev, Matthias Pätzold \\ Faculty of Engineering and Science, University of Agder \\ Correspondence: Matthias Pätzold, matthias.paetzold@uia.no \\ Manuscript communication: received 23 March 2012, accepted 3 April 2012
}

\begin{abstract}
In this paper, we present an improved version of the iterative nonlinear least square approximation (INLSA) method for designing measurement-based single-input single-output (SISO) wideband channel simulators. The proposed method aims to fit the time-frequency correlation function (TFCF) of the simulation model to that of a measured channel. The parameters of the simulation model are determined iteratively by minimizing the Frobenius norm, which serves as a measure for the fitting error. In contrast to the original INLSA method, the proposed approach provides a unique optimized set of model parameters, which guarantees a quasi-perfect fitting with respect to the TFCF. We analyze the performance of the proposed method in terms of the goodness of fit to the measured data. The investigations will be carried out with respect to the TFCF and the scattering function. We demonstrate that the proposed approach is a powerful tool for the design of measurement-based wideband channel simulators, which are important for the performance evaluation of mobile communication systems under real-world propagation conditions.
\end{abstract}

Keywords- Mobile fading channels, measurement-based channel models, channel simulators, single-input single-output, time-frequency correlation function, iterative parametrization.

This paper was presented in part at the 4th International Conference on Advanced Technologies for Communications (ATC 2011), Danang, Vietnam.

\section{INTRODUCTION}

The reliability and the maximum achievable data rate of a communication system are heavily dependent on the characteristics of the propagation channel. Hence, during the design and test phase, it is of utmost importance to have a realistic channel model to begin with. A lot of research attention has been devoted to the development of channel models the statistics of which matches very closely that of a real propagation environment. The most recent comprehensive survey on existing modeling approaches is reported in [1], [2].

In general, sophisticated channel models are based on wideband scenarios, which incorporate the frequency selectivity of the physical channel. A widely used approach to develop wideband channel models is to represent the time-variant transfer function by a finite sum of multipath propagation components [3], [4]. The efficient development of wideband channel models relies on a sophisticated method for determining the primary model parameters, which are the path gains, Doppler frequencies, and propagation delays. Hence, advanced and efficient parameter computation methods are required to accurately estimate these model parameters from the measured channels. In last couple of decades, various high resolution parameter estimation methods have been proposed to derive the reference models [5]-[8]. These methods can also be used as parameter computation techniques for designing measurement-based channel simulators. In [5], e.g., the multiple signal classification (MUSIC) algorithm has been applied for delay estimation. A joint angle and delay estimation (JADE) technique is proposed in [9]. A direction-of-arrival estimation method using the estimation of signal parameters via rotational invariance techniques (ESPRIT) algorithm has been discussed in [6]. The unitary ESPRIT has been proposed in [10] to improve the estimation accuracy of the ESPRIT algorithm. The ESPRIT method has further been extended in [11] to enable a joint delay and azimuth estimation, while the unitary ESPRIT technique has been used in [12] to perform a joint azimuth and elevation estimation. An application of the unitary ESPRIT algorithm to wide-band channel measurements is presented in [13].Owing to the high implementation complexity of the aforementioned methods, maximum-likelihood-based algorithms are preferable for solving the channel parameter estimation problem. These include the expectation maximization [7] and the space alternating generalized expectation maximization (SAGE) [8] algorithms. Particularly the SAGE algorithm is widely used for channel parameter estimation due to its excellent performance in most scenarios. However, the SAGE algorithm often requires a large amount 
of specular components, which makes the estimation process relatively complex. In [14], the authors reported a initialization sensitivity problem of the SAGE algorithm. Several approaches have been proposed in [14]-[17] to mitigate the drawbacks of this algorithm. In [17], the authors proposed the iterative nonlinear least square approximation (INLSA) method for the design of measurement-based wideband channel simulators. The authors showed that the INLSA algorithm provides a good match to empirical data by keeping the complexity lower than that of the SAGE algorithm. The INLSA algorithm has further been refined in [18].

In this paper, we extend the INLSA method to allow a joint estimation of the primary model parameters. The INLSA algorithm is based on the expectation maximization approach [7]. In order to reduce the computational cost, the INLSA algorithm computes the maximumlikelihood estimates of the channel model parameters iteratively. The iterative approach, however, may lead to a poor modeling of the TFCF of the measured channel at the origin. The value at the origin of the TFCF is of crucial importance for computing the characteristic quantities describing the statistics of the fading channel, such as the probability density function of the envelope, the level-crossing rate, and the average duration of fades. The original INLSA method [17] offers two alternatives for optimizing the simulation model parameters. The first alternative computes the path gains together with the Doppler frequencies by modeling the measured temporal autocorrelation function (TACF), and then computes the propagation delays in a second step from the frequency correlation function (FCF) of the measured channel. This variant of the original INLSA algorithm will be called the INLSA-T-F method. The second alternative computes first the path gains jointly with the propagation delays by modeling the measured FCF, whereas the Doppler frequencies are obtained in a second step from the TACF of the measured channel. The second variant of the INLSA algorithm will be referred to as the INLSA-F-T method. These kinds of separate optimizations of the simulation model parameters may lead to erroneous parameter estimations when evaluating the resulting channel simulator with respect to the TFCF. Moreover, the INLSAT-F and INLSA-F-T approaches result in different sets of estimated parameters for identical measured data.

In order to overcome the aforementioned drawbacks of the original INLSA algorithm, the proposed method computes the simulation model parameters jointly by fitting the TFCF of the simulation model to that of the measured channel. The proposed method is called INLSA-TF emphasizing the joint modeling of both the temporal and frequency correlation functions of real-world channels. The proposed algorithm includes additional error compensation steps in the iterative estimation procedure to guarantee a perfect fitting at the origin of the measured TFCF. As a result, the proposed method provides a unique set of optimized model parameters while maintaining a perfect fitting at the origin. In order to demonstrate the performance of the proposed INLSA-TF method, we implemented the algorithm in MATLAB ${ }^{\circledR}$ and compared the new approach with the two known variants of the original INLSA method. We focused on a number of issues which are of particular interest in channel modeling, such as the fitting accuracy with respect to the most important correlation functions and the scattering function of the measured channel. In addition to this, we studied the performance of the measurement-based simulation model as a function of the selected number of propagation paths.

This paper is structured as follows. In Section 2, we introduce the wideband channel simulation model. In Section 3, the parameter estimation problem is formulated. Section 4 describes the proposed parameter computation method. The application to measurement data is presented in Section 5. Finally, Section 6 concludes this paper.

\section{The Wideband Channel Simulation MODEL}

In this section, we describe the simulation model on which we apply the parameter computation method presented in this work. We also derive the TFCF of the simulation model under the assumption that the autocorrelation ergodicity conditions are fulfilled.

An important objective for the design of channel simulation models is to guarantee that their statistical properties are as close as possible to real-world channels. When using a measurement-based approach, we have to consider that every measurement provides only propagation area specific information for a short period of time. This means we have to select a proper channel model the parameters of which can be estimated from given snapshot measurements. For this reason, we adopt the widely accepted model from [19], which represents the time-variant frequency response (TVFR) of multipath fading channel as a superposition of a finite number of $N$ propagation paths in the form

$$
H\left(f^{\prime}, t\right)=\sum_{n=1}^{N} c_{n} e^{j\left(2 \pi f_{n} t-2 \pi f^{\prime} \tau_{n}^{\prime}+\theta_{n}\right)},
$$

where $t$ and $f^{\prime}$ are the time and frequency variables, respectively. Each propagation path is characterized by its path gain $c_{n}$, propagation delay $\tau_{n}^{\prime}$, Doppler frequency $f_{n}$, and the phase shift $\theta_{n}$. The number of propagation paths $N$ determines the accuracy as well as the complexity of the simulation model. We assume that the elements of the set $\left\{\theta_{n}\right\}$ are independent and identically distributed (i.i.d.) random variables, each having a uniform distribution in the interval $[0,2 \pi)$. Our aim is to determine the remaining sets of parameters $\left\{c_{n}\right\},\left\{f_{n}\right\}$, and $\left\{\tau_{n}^{\prime}\right\}$ of the channel simulation model described in (1) such that the simulation model has almost the same statistical characteristics as a given measured channel. For our analysis, it will suffice to consider the TVFR $H\left(f^{\prime}, t\right)$ at discrete frequencies $f_{m}^{\prime}=-B / 2+m \Delta f^{\prime} \in[-B / 2, B / 2], m=0,1, \ldots, M-1$, and at discrete time instances $t_{k}=k \Delta t \in[0, T], k=$ 
$0,1, \ldots, K-1$, where $B$ and $T$ denote the measured frequency bandwidth and the observation time interval, respectively. The time sampling interval $\Delta t$ and the frequency sampling interval $\Delta f^{\prime}$ are characteristic quantities of the channel sounder used to collect the measurement data. Consequently, the TVFR $H\left(f^{\prime}, t\right)$ can be represented as a discrete TVFR $H\left[f_{m}^{\prime}, t_{k}\right]$. For simplicity's sake, it is assumed that the parameters of the simulation model described by (1) fulfill the following conditions

$$
\begin{aligned}
& f_{n} \neq f_{m}, \tau_{n}^{\prime} \neq \tau_{m}^{\prime}, \quad \forall n \neq m, \\
& E\left\{e^{j\left(\theta_{n}-\theta_{m}\right)}\right\}=0, \quad \forall n \neq m,
\end{aligned}
$$

where $E\{\cdot\}$ represents the statistical averaging operator. The condition (3) is always fulfilled as $\left\{\theta_{n}\right\}$ are i.i.d. random variables, which are uniformly distributed over the interval $[0,2 \pi]$. As a result of the imposed constraints (2) and (3), the channel model in (1) is autocorrelation ergodic with respect to time and frequency [20]. In this case, the discrete TFCF $R\left[v_{p}^{\prime}, \tau_{q}\right]$ of the simulation model can be deduced from a single realization of the discrete TVFR $H\left[f_{m}^{\prime}, t_{k}\right]$ as follows

$$
\begin{aligned}
R\left[v_{p}^{\prime}, \tau_{q}\right] & =\left\langle H\left[f_{m}^{\prime}, t_{k}\right] H^{*}\left[f_{m}^{\prime}+v_{p}^{\prime}, t_{k}+\tau_{q}\right]\right\rangle \\
& =\sum_{n=1}^{N} c_{n}^{2} e^{j 2 \pi\left(\tau_{n}^{\prime} v_{p}^{\prime}-f_{n} \tau_{q}\right)}
\end{aligned}
$$

where $\tau_{q}=0, \Delta t, \ldots,(K-1) \Delta t$ and $v_{p}^{\prime}=$ $0, \Delta f^{\prime}, \ldots,(M-1) \Delta f^{\prime}$. The notation $\langle\cdot\rangle$ denotes averaging over time and frequency.

The preceding equation shows that $R\left[v_{p}^{\prime}, \tau_{q}\right]$ depends on the number of paths $N$, path gains $c_{n}$, propagation delays $\tau_{n}^{\prime}$, and Doppler frequencies $f_{n}$, but not on the phase shifts $\theta_{n}$. The TACF $r_{t_{k}}\left[\tau_{q}\right]$ is obtained from the TFCF $R\left[v_{p}^{\prime}, \tau_{q}\right]$ by setting $v_{p}^{\prime}$ to zero, i.e.,

$$
r_{t_{k}}\left[\tau_{q}\right]=R\left[0, \tau_{q}\right]=\sum_{n=1}^{N} c_{n}^{2} e^{-j 2 \pi f_{n} \tau_{q}} .
$$

The FCF $r_{f_{m}^{\prime}}\left[v_{p}^{\prime}\right]$ of the simulation model can be determined from the TFCF $R\left[v_{p}^{\prime}, \tau_{q}\right]$ by setting $\tau_{q}$ to zero, i.e.,

$$
r_{f_{m}^{\prime}}\left[v_{p}^{\prime}\right]=R\left[v_{p}^{\prime}, 0\right]=\sum_{n=1}^{N} c_{n}^{2} e^{j 2 \pi \tau_{n}^{\prime} v_{p}^{\prime}} .
$$

For mathematical convenience, the discrete TFCF $R\left[v_{p}^{\prime}, \tau_{q}\right]$ will be represented by an $M \times K$ timefrequency correlation matrix (TFCM) $\mathbf{R}$. It is straightforward to show that the TFCM $\mathbf{R}$ can be formulated as

$$
\mathbf{R}=\sum_{n=1}^{N} c_{n}^{2} \mathbf{P}_{n}
$$

where $\mathbf{P}_{n}$ is an $M \times K$ matrix and is defined as follows:

$$
\mathbf{P}_{n}=\left(\begin{array}{ccc}
\sum_{n=1}^{N} e^{j 2 \pi\left(\tau_{n}^{\prime} v_{0}^{\prime}-f_{n} \tau_{0}\right)} & \cdots & \sum_{n=1}^{N} e^{j 2 \pi\left(\tau_{n}^{\prime} v_{0}^{\prime}-f_{n} \tau_{K-1}\right)} \\
\vdots & \ddots & \vdots \\
\sum_{n=1}^{N} e^{j 2 \pi\left(\tau_{n}^{\prime} v_{M-1}^{\prime}-f_{n} \tau_{0}\right)} & \cdots & \sum_{n=1}^{N} e^{j 2 \pi\left(\tau_{n}^{\prime} v_{M-1}^{\prime}-f_{n} \tau_{K-1}\right)}
\end{array}\right)
$$

\section{Problem Formulation}

In this section, we describe the measured channel and discuss its correlation properties. We also formulate the problem of determining the simulation model parameters.

Let $\check{H}\left(f^{\prime}, t\right)$ denote the TVFR of a real-world channel measured at discrete frequencies $f_{m}$, and at discrete time instances $t_{k}$. In this case, the TVFR $\breve{H}\left(f^{\prime}, t\right)$ can be represented in the form of a discrete function, which will be denoted by $\check{H}\left[f_{m}^{\prime}, t_{k}\right]$. Further, we assume that the measured TVFR $H\left[f_{m}^{\prime}, t_{k}\right]$ is wide-sense stationary [21] and autocorrelation ergodic with respect to time and frequency. This allows us to obtain the discrete TFCF $\check{R}\left[v_{p}^{\prime}, \tau_{q}\right]$ of the measured channel from $\check{H}\left[f_{m}^{\prime}, t_{k}\right]$ by averaging over time and frequency. Thus,

$$
\check{R}\left[v_{p}^{\prime}, \tau_{q}\right]=\frac{1}{K M} \sum_{k=0}^{K-1} \sum_{m=0}^{M-1} \check{H}\left[f_{m}^{\prime}, t_{k}\right] \check{H}^{*}\left[f_{m}^{\prime}+v_{p}^{\prime}, t_{k}+\tau_{q}\right] .
$$

The TACF $\breve{r}_{t_{k}}\left[\tau_{q}\right]$ and the FCF $\check{r}_{f_{m}^{\prime}}\left[v_{p}^{\prime}\right]$ of the measured channel are obtained from $\check{R}\left[v_{p}^{\prime}, \tau_{q}\right]$ in (9) by setting $v_{p}^{\prime}=0$ and $\tau_{q}=0$, respectively, i.e., $\check{r}_{t_{k}}\left[\tau_{q}\right]=\check{R}\left[0, \tau_{q}\right]$ and $\check{r}_{f_{m}^{\prime}}\left[v_{p}^{\prime}\right]=\check{R}\left[v_{p}^{\prime}, 0\right]$. For mathematical convenience, we represent the discrete TFCF $\check{H}\left[f_{m}^{\prime}, t_{k}\right]$ by an $M \times K$ TFCM ̌r.

With reference to the simulation model described by (1), the problem at hand is to determine the set of parameters $\mathcal{P}=\left\{N, c_{n}, f_{n}, \tau_{n}^{\prime}\right\}$ in such a way that the statistical properties of the simulation model match those of the measured channel. Here, the desired statistical properties are described by the TFCM $\check{\mathbf{R}}$ of the measured channel. The problem of determining the set of parameters $\mathcal{P}$ can now be formulated as follows:

Given is the measured discrete TVFR $\check{H}\left[f_{m}^{\prime}, t_{k}\right]$ of a real-world channel. Determine the set of parameters $\mathcal{P}=$ $\left\{N, c_{n}, f_{n}, \tau_{n}^{\prime}\right\}$ of the channel simulation model described by (1), such that the TFCM $\mathbf{R}$ of the simulation model is as close as possible (in the Frobenius norm sense) to the TFCM $\check{\mathbf{R}}$ of the measured channel, i.e.,

$$
\mathcal{P}=\arg \min _{\mathcal{P}}\|\check{\mathbf{R}}-\mathbf{R}\|_{F}
$$

where $\|\cdot\|_{F}$ denotes the Frobenius norm.

As it was pointed out in [17], the problem formulation in (10) is valid if the measured channel is autocorrelation ergodic.

\section{The Proposed Parameter Computation MeTHOD}

This section focuses on the description of the INLSA-TF algorithm proposed for computing the parameters of the measurement-based channel simulator. By referring to the problem formulation discussed in Section 3 and assuming that the discrete TFCM $\check{\mathbf{R}}$ of the measured channel is known, we measure the model error between the measured channel and the simulation model by

$$
E(\mathcal{P})=\left\|\check{\mathbf{R}}-\sum_{n=1}^{N} c_{n}^{2} \mathbf{P}_{n}\right\|_{F} .
$$


The objective is to minimize the Frobenius norm in (11), such that the TFCM $\mathbf{R}$ of the simulation is fitted as close as possible to the given TFCM $\check{\mathbf{R}}$ of the measured channel. Owing to the complexity of this optimization problem, the implementation of the maximum-likelihood estimation of model parameters that minimizes the error in (11) is intractable. Therefore, the proposed method utilizes the iterative approach to optimize the simulation model parameters. The iterative parameter computation method starts with $N=1$ and arbitrarily chosen initial values for $f_{1}^{(0)}$ and $\tau_{1}^{\prime(0)}$. At every iteration $i(i=0,1,2, \ldots)$, the algorithm computes the complete set of model parameters $\mathcal{P}$. The parameter computation procedure is carried out separately for each path $l(l=1,2, \ldots, N)$ by using the auxiliary error matrix as follows:

$$
\begin{aligned}
\mathbf{Y}_{l}^{(i)} & =\check{\mathbf{R}}-\sum_{n=1, n \neq l}^{N}\left(c_{n}^{(i)}\right)^{2} \mathbf{P}_{n}^{(i)} \\
c_{l}^{(i+1)} & =\arg \min _{c_{l}}\left\|\mathbf{Y}_{l}^{(i)}-c_{l}^{2} \mathbf{P}_{l}^{(i)}\right\|_{F} \\
f_{l}^{(i+1)} & =\arg \min _{f_{l}}\left\|\mathbf{Y}_{l}^{(i)}-\left(c_{l}^{(i+1)}\right)^{2} \mathbf{P}_{l}^{(i)}\right\|_{F} \\
\tau_{l}^{(i+1)} & =\arg \min _{\tau_{l}^{\prime}}\left\|\mathbf{Y}_{l}^{(i)}-\left(c_{l}^{(i+1)}\right)^{2} \mathbf{P}_{l}^{(i+1)}\right\|_{F}
\end{aligned}
$$

where the $M \times K$ matrix $\mathbf{P}_{l}^{(i+1)}$ is recomputed by substituting the optimized values of $f_{l}^{(i+1)}$ in (8). In this algorithm, the iterative computation of the set of parameters $\mathcal{P}$ continues as long as the relative change in the model error $E(\mathcal{P})$ is larger than a predefined threshold level $\varepsilon$. When the threshold level $\varepsilon$ is reached, the iteration stops, and the number of propagation paths $N$ is increased by one, i.e., $N+1 \rightarrow N$. The initial values of $c_{N}^{(0)}, f_{N}^{(0)}$, and $\tau_{N}^{\prime(0)}$ are set to zero. After this, the iterative parameter computation procedure is carried out again starting from (12). This process is repeated until no perceptible progress can be made by increasing $N$ or the maximum number of paths $N_{\max }$ is reached.

It is straightforward to show that (13) results in a minimum if $c_{l}^{(i+1)}$ is computed as

$$
c_{l}^{(i+1)}=\sqrt{\frac{\Re\left\{\mathbf{y}_{l}^{(i)}\right\}^{T} \Re\left\{\mathbf{p}_{l}^{(i)}\right\}+\Im\left\{\mathbf{y}_{l}^{(i)}\right\}^{T} \Im\left\{\mathbf{p}_{l}^{(i)}\right\}}{\left(\mathbf{p}_{l}^{(i)}\right)^{H} \mathbf{p}_{l}^{(i)}}},
$$

where the operators $\{\cdot\}^{T}$ and $\{\cdot\}^{H}$ denote the transpose and complex-conjugate transpose, respectively. The symbol $\mathbf{y}_{l}^{(i)}$ refers to a column vector formed by mapping the elements in $\mathbf{Y}_{l}^{(i)}$ such that all columns in $\mathbf{Y}_{l}^{(i)}$ are stacked on top of each other. Analogously, the symbol $\mathbf{p}_{l}^{(i)}$ denotes a column vector, which is formed by applying the same mapping scheme to $\mathbf{P}_{l}^{(i)}$. However, in order to guarantee a perfect fitting at the origin $v_{p}^{\prime}=0$ and $\tau_{q}=0$ of the TFCF $\check{R}\left[v_{p}^{\prime}, \tau_{q}\right]$, we replace the optimization step in (13) by

$$
c_{l}^{(i+1)}= \begin{cases}\sqrt{\frac{\Re\left\{\mathbf{y}_{l}^{(i)}\right\}^{T} \Re\left\{\mathbf{p}_{l}^{(i)}\right\}+\Im\left\{\mathbf{y}_{l}^{(i)}\right\}^{T} \Im\left\{\mathbf{p}_{l}^{(i)}\right\}}{\left(\mathbf{p}_{l}^{(i)}\right)^{H} \mathbf{p}_{l}^{(i)}},} & l \neq N \\ \check{R}[0,0]-\sum_{l=1}^{N-1}\left(c_{l}^{(i+1)}\right)^{2}, & l=N .\end{cases}
$$

The preceding equation implies that the $c_{N}$ is computed such that exact fitting of $\tilde{R}\left[v_{p}^{\prime}, \tau_{q}\right]$ is always preserved if $v_{p}^{\prime}=0$ and $\tau_{q}=0$.

The procedure above is coined INLSA-TF. It should be pointed out here that when using the INLSA-TF method, the path gains are optimized in combination with the corresponding Doppler frequencies and propagation delays, which result in an unambiguous and unique triple $\left(c_{n}, f_{n}, \tau_{n}^{\prime}\right)$ for each propagation path $n=1,2, \ldots, N$. This is in contrast to the INLSA-T-F and INLSA-F-T methods for which we obtain only the unique tuples $\left(c_{n}, f_{n}\right)$ and $\left(c_{n}, \tau_{n}^{\prime}\right)$, respectively. Since the optimization process in the proposed method is carried out by modeling the TFCF of the measured channel, the INLSA-TF method provides an excellent performance when evaluating the channel simulator with respect to the TFCF. As we will see, the INLSA-T-F and INLSAF-T methods cannot provide an accurate modeling of the TFCF of the measured channel, especially close to the origin. The results of a comparative study of these three approaches are demonstrated in the next section.

\section{Application to Measurement Data}

In this section, we demonstrate the usefulness and the performance of the proposed INLSA-TF algorithm. To accomplish these two objectives, we used measurement data collected in an urban area in the $2.1 \mathrm{GHz}$ frequency band. In the measurement campaign, the transmitter antenna was mounted on a trolley and acted as a mobile part. The receiver antenna was stationary and mounted on the roof of a building at a height of $20 \mathrm{~m}$. The performed measurements were double-directional multipath measurements conducted using a wideband channel sounder. The key parameters of the measurement equipment are:

- time sampling interval: $\Delta t=0.02 \mathrm{~s}$,

- frequency sampling interval: $\Delta f^{\prime}=195 \mathrm{kHz}$,

- bandwidth: $B=100 \mathrm{MHz}$,

- observation time interval: $T=0.5 \mathrm{~s}$.

For comparative purposes, we first demonstrate the channel estimation capabilities of the proposed INLSATF algorithm and the two original variants of the INLSA method using the same measurement data. The threshold level $\epsilon$ of the proposed algorithm was empirically set to $\epsilon=0.01$ in all cases. Figure 1 illustrates the normalized residual error norm $\|\breve{R}-R\|_{F} /\|\check{R}\|_{F}$ versus the number of propagation paths $N$.

As can be seen from the results shown in this figure, the proposed INLSA-TF method performs significantly better than the two original INLSA approaches. The different performances of the INLSA-T-F and INLSAF-T originate from the fact that the estimated sets of 


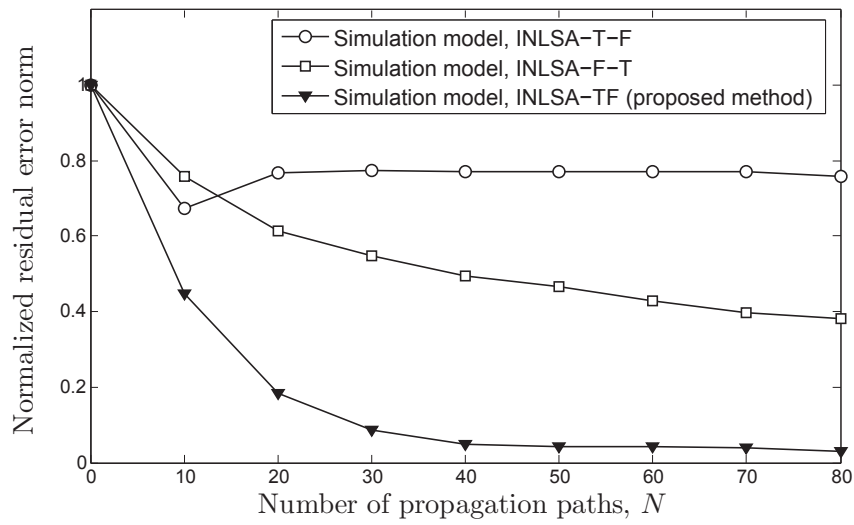

Figure 1. Normalized residual error norm $\|\check{R}-R\|_{F} /\|\check{R}\|_{F}$ versus the number of propagation paths $N$.

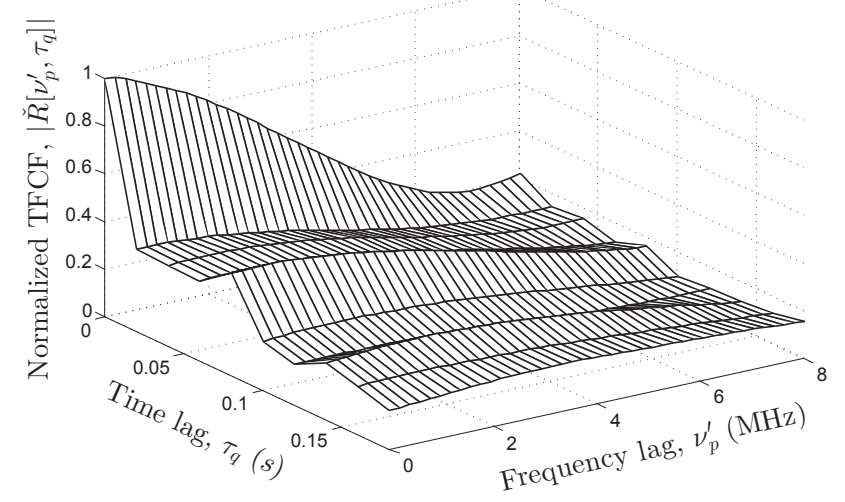

Figure 2. Absolute value of the normalized TFCF $\left|\check{R}\left[v_{p}^{\prime}, \tau_{q}\right]\right|$ of the measured channel.

model parameters are not identical when these methods are used. As an aside, we want to mention that the INLSA-TF algorithm as well as the two original versions (INLSA-T-F and INLSA-F-T) are initialization insensitive. This means that, for any chosen initial values of the Doppler frequencies and path delays, we always get the same graph for the normalized residual error norm. We also realize from the inspection of Figure 1 that the normalized residual error norm associated with the INLSA-TF method is approximately $10 \%$ if $N=30$, whereas the error norm drops below $3 \%$ if $N=80$. By referring to these results, the number of propagation paths $N_{\max }=80$ is considered to be sufficient and adopted for further simulations as a stopping criterion in the iterative optimization procedure.

The important difference between the proposed and the original INLSA method is that the former one aims to model the TFCF of real-world channels, while the latter one focuses only on the behavior of the TFCF along the principal axes, meaning the TACF and the FCF. In order to demonstrate this difference, we have plotted the TFCFs of the measured channel and the simulation model by using the INLSA-TF and the INLSA-T-F in Figures 2, 3, and 4, respectively. From Figures $2-4$, we can realize that the INLSA-TF method performs significantly better than the original INLSAT-F. It is readily apparent from comparing the results in

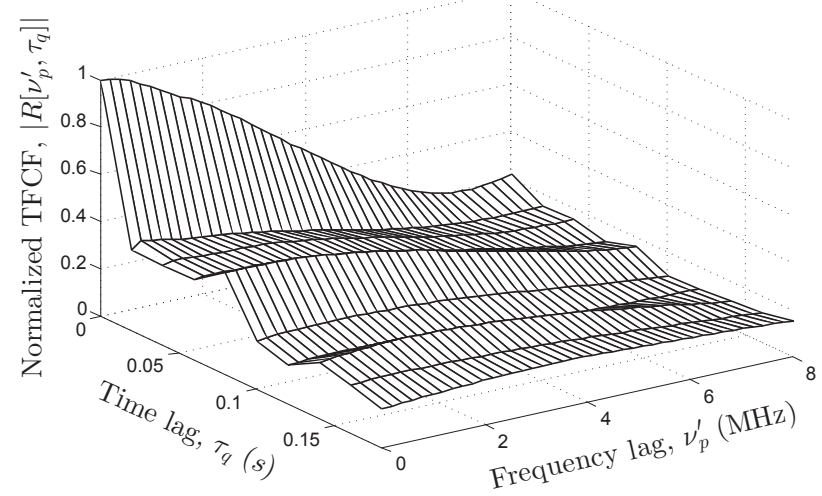

Figure 3. Absolute value of the normalized TFCF $\left|R\left[v_{p}^{\prime}, \tau_{q}\right]\right|$ of the simulation model with the parameters estimated by using the proposed INLSA-TF method.

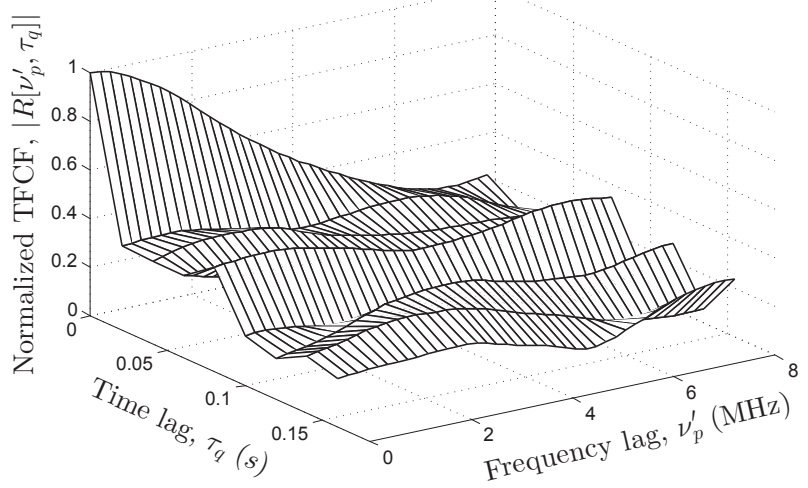

Figure 4. Absolute value of the normalized TFCF $\left|R\left[v_{p}^{\prime}, \tau_{q}\right]\right|$ of the simulation model with the parameters estimated by using the INLSAT-F method.

Figure 2 and Figure 4 that the main focus of the original INLSA algorithm falls only on the TACF and the FCF.

As mentioned in Section 1, the separate parameter optimization approach implemented in the original INLSA algorithm may lead to incorrect estimations of the simulation model's parameters. To highlight this problem, we have illustrated the performance of the two variants of the original INLSA method in Figures 5 and 6 . As can be seen in those figures, if the INLSA-F-T approach is used, the difference between the TACF curves of the simulation model and the measured channel is significant, whereas the FCF of the simulation model closely matches the FCF of the measured channel. However, there is a significant gap between the FCFs close to the origin. The discrepancy between the FCFs of the simulation model and the measured channel is relatively small if the INLSA-TF method is applied. This characteristic feature of the INLSA-F-T method close to the origin is a result of the used iterative approach for simplifying the maximumlikelihood optimization problem. We can also conclude from the inspection of Figures 5 and 6 that the TACF and the FCF of the simulation model are well fitted to the corresponding correlation functions of the measured channel if the simulation model's parameters are computed by using the proposed INLSA-TF method. 


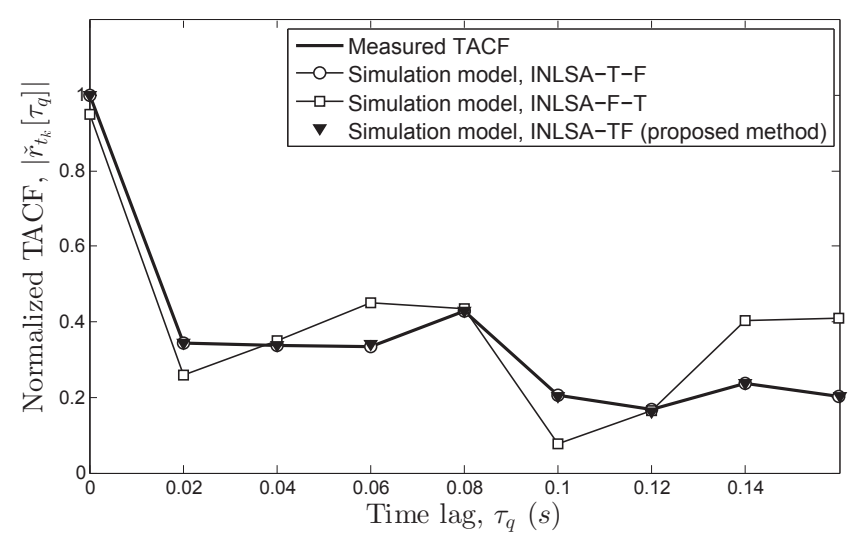

Figure 5. Absolute value of the normalized TACFs $\left|\check{r}_{t_{k}}\left[\tau_{q}\right]\right|$ (measured channel) and $\left|r_{t_{k}}\left[\tau_{q}\right]\right|$ (simulation model) for different parameter computation methods.

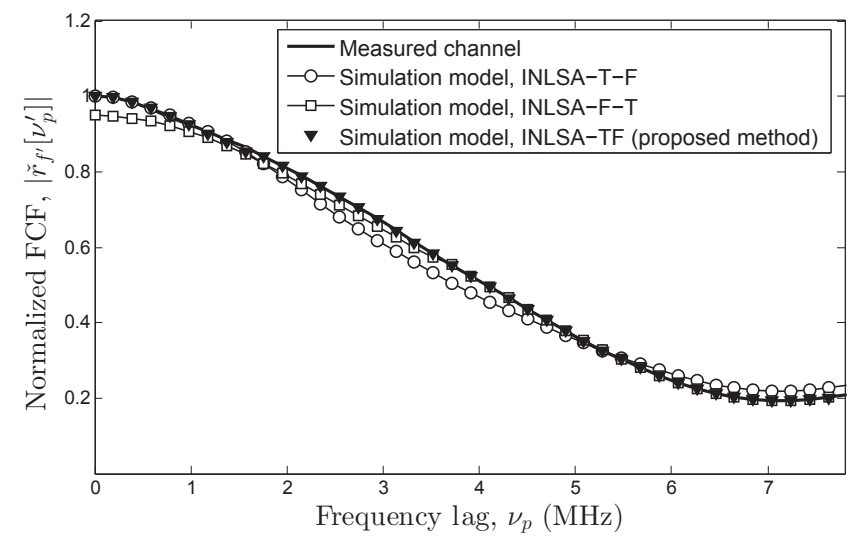

Figure 6. Absolute value of the normalized FCFs $\left|\check{r}_{f_{m}^{\prime}}\left[v_{p}^{\prime}\right]\right|$ (measured channel) and $\left|r_{f_{m}^{\prime}}\left[v_{p}^{\prime}\right]\right|$ (simulation model) for different parameter computation methods.

Impressively, the proposed method provides an exact correspondence between the correlation functions of the simulation model and the measured channel at the origin, as it was expected from (17).

In our performance analysis, we have also incorporated the study of the scattering function $S\left(\tau^{\prime}, f\right)$ of the simulation model, which is obtained by applying the two-dimensional discrete Fourier transform to the TFCF $R\left[v_{p}^{\prime}, \tau_{q}\right]$. In Figure 7 , we have depicted a comparison between the scattering function of the measured channel and that of the simulation model designed by using the proposed INLSA-TF algorithm. The corresponding results obtained by applying the original INLSA method are shown in Figure 8. An excellent agreement between the scattering functions of the measured channel and the simulation model is observed if the proposed INLSA-TF method is used (see Figure 7). The results presented in Figure 8 demonstrate the poor performance of the INLSA-T-F and the INLSAF-T methods in terms of modeling the scattering function of the measured channel.

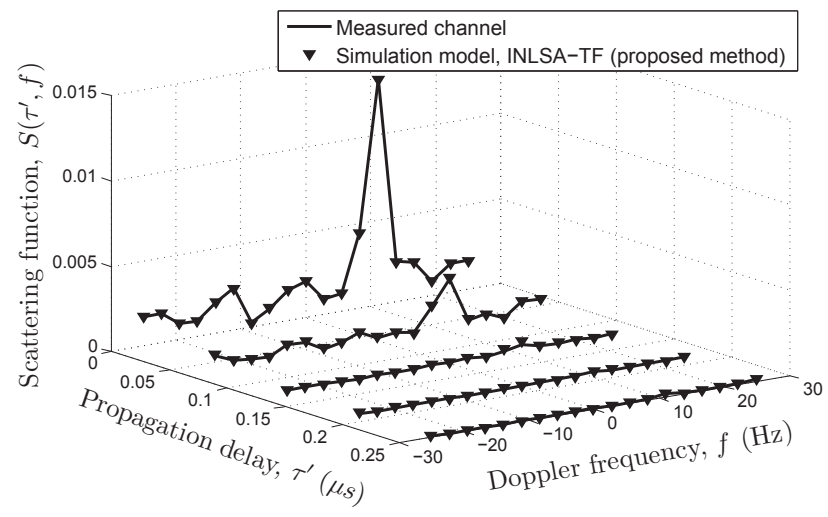

Figure 7. Comparison between the scattering functions $\check{S}\left(\tau^{\prime}, f\right)$ (measured channel) and $S\left(\tau^{\prime}, f\right)$ (simulation model) designed by using the INLSA-TF method.

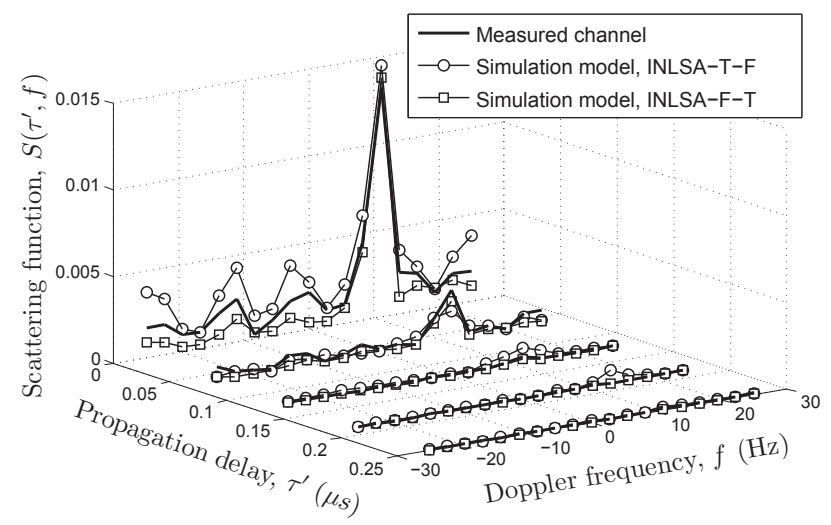

Figure 8. Comparison between the scattering functions $\check{S}\left(\tau^{\prime}, f\right)$ (measured channel) and $S\left(\tau^{\prime}, f\right)$ (simulation model) designed by using the INLSA method.

\section{Summary and Conclusions}

In this paper, we introduced the INLSA-TF method for designing measurement-based wideband channel simulators. The proposed method is an extension of the INLSA method. The INLSA-TF method aims to iteratively compute an optimized set of parameters describing the channel simulator by modeling the empirical TFCF of the given measurement data.

A performance analysis of the proposed method has been conducted by applying the INLSA-TF algorithm to measurement data collected in an urban environment. We have compared the correlation properties of the simulation model with those of the measured channel. A comparison has also been made with respect to the scattering function.

The obtained results demonstrated that the INLSATF algorithm precisely estimates the simulation model parameters and provides an excellent fitting to the statistics of real-world channels. Finally, it has been shown that the INLSA-TF method significantly outperforms the two original versions. Owing to the low complexity and an excellent performance, the INLSATF method can be considered as a powerful tool for synthesizing real-world channels and can therefore be used for the performance analysis of mobile communication systems under real-world propagation conditions. 


\section{ACKNOWLEDGMENT}

The authors would like to thank Elektrobit Corporation for the kindly provided measurement data.

\section{REFERENCES}

[1] H. Krim and M. Viberg, "Two decades of array signal processing research: The parametric approach," IEEE Signal Processing Magazine, vol. 13, pp. 67-94, July 1996.

[2] P. Almers, E. Bonek, A. Burr, N. Czink, M. Debbah, V. DegliEsposti, H. Hofstetter, P. Kyösti, D. Laurenson, G. Matz, A. F. Molisch, C. Oestges, and H. Özcelik, "Survey of channel and radio propagation models for wireless MIMO systems," EURASIP Journal on Wireless Communications and Networking, vol. 2007, pp. Article ID: 19070, 19 pages, 2007.

[3] R. S. Thomä, D. Hampicke, M. Landmann, A. Richter, and G. Sommerkorn, "Measurement-based parametric channel modelling (MBPCM)," in Int. Conf. on Electromagnetics in Advanced Applications, (ICEAA 2003), Torino, Italia, Sept. 2003.

[4] M. Pätzold, Mobile Radio Channels, 2nd ed. Chichester: John Wiley \& Sons, 2011.

[5] R. Schmidt, "Multiple emitter location and signal parameter estimation," IEEE Transactions on Antennas and Propagation, vol. 34, no. 3, pp. 276-280, Mar. 1986.

[6] R. Roy and T. Kailath, "ESPRIT-estimation of signal parameters via rotational invariance techniques," IEEE Transactions on Acoustics, Speech, and Signal Processing, vol. 37, no. 7, pp. 984-995, 1989.

[7] M. Feder and E. Weinstein, "Parameter estimation of superimposed signals using the EM algorithm," IEEE Transactions on Acoustics, Speech, and Signal Processing, vol. 36, no. 4, pp. 477489,1988

[8] B. H. Fleury, M. Tschudin, R. Heddergott, D. Dahlhaus, and K. Ingeman Pedersen, "Channel parameter estimation in mobile radio environments using the SAGE algorithm," IEEE Journal on Selected Areas in Communications, vol. 17, no. 3, pp. 434-450, 1999.

[9] M. C. Vanderveen, A.-J. Van der Veen, and A. Paulraj, "Estimation of multipath parameters in wireless communications," vol. 46, no. 3, pp. 682-690, May 1998.

[10] M. Haardt and J. A. Nossek, "Unitary esprit: how to obtain increased estimation accuracy with a reduced computational burden," IEEE Transactions on Signal Processing, vol. 43, no. 5, pp. 1232-1242, May 1995.

[11] A. van der Veen, M. Vanderveen, and A. Paulraj, "Joint angle and delay estimation using shift-invariance properties," IEEE Signal Processing Letters, vol. 4, pp. 142-145, May 1997.

[12] M. Zoltowski, M. Haardt, and C. Mathews, "Closed-form 2$\mathrm{D}$ angle estimation with rectangular arrays in element space or beamspace via unitary ESPRIT," IEEE Transactions on Signal Processing, vol. 44, pp. 316-328, Feb. 1996.

[13] J. Fuhl, J.-P. Rossi, and E. Bonek, "High-resolution 3-D directionof-arrival determination for urban mobile radio," IEEE Transactions on Antennas and Propagation, vol. 45, pp. 672-682, Apr. 1997.

[14] W. Li and Q. Ni, "Joint channel parameter estimation using evolutionary algorithm," in Proc. 2010 IEEE International Conference on Communications (ICC 2010), Cape Town, South Africa, May 2010, pp. 1-6.

[15] W. Li, W. Yao, and P. J. Duffett-Smith, "Improving the SAGE algorithm with adaptive partial interference cancellation," in Proc. IEEE 13th Digital Signal Processing Workshop and 5th IEEE Signal Processing Education Workshop (DSP/SPE 2009), 2009, pp. 404-409.

[16] C. B. Ribeiro, E. Ollila, and V. Koivunen, "Stochastic maximum likelihood method for propagation parameter estimation," in Proc. 15th IEEE Int. Symp. Personal, Indoor and Mobile Radio Communications (PIMRC 2004), vol. 3, 2004, pp. 1839-1843.

[17] D. Umansky and M. Pätzold, "Design of measurement-based wideband mobile radio channel simulators," in Proc. 4th IEEE International Symposium on Wireless Communication Systems (ISWCS 2007), Trondheim, Norway, Oct. 2007, pp. 229-235.

[18] A. Fayziyev and M. Pätzold, "An improved iterative nonlinear least square approximation method for the design of measurement-based wideband mobile radio channel simulators," in Proc. Int. Conf. Advanced Technologies for Communications (ATC 2011), Danang, Vietnam, Apr. 2011, pp. 106-111.

[19] G. D. Durgin, Space-Time Wireless Channels. NJ: Prentice Hall, 2003.
[20] C. A. Gutiérrez, A. Meléndez, A. Sandoval, and H. Rodriguez, "On the autocorrelation ergodic properties of sum-of-cisoids Rayleigh fading channel simulators," in Proc. European Wireless (EW 2011), Vienna, Austria, Apr. 2011, pp. 1-6.

[21] A. Papoulis and S. Pillai, Probability, Random Variables and Stochastic Processes. New York: McGraw-Hill, 2004.

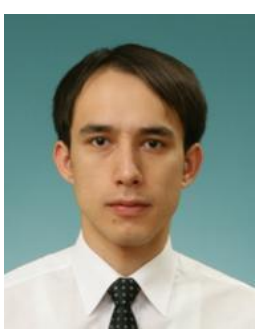

Akmal Fayziyev was born in Tashkent Uzbekistan, in 1984. He received his Bachelor's degree in Telecommunications from Tashkent University of Information Technologies, Uzbekistan, in 2005. In August 2007, he received the Master of Science (M.Sc.) degree in Engineering from Yeungnam University, South Korea. From 2007 to 2009, he worked as a core network department engineer at UCell, a cellular GMS/3G operator in Uzbekistan. Since July 2009, he is working on his $\mathrm{PhD}$ thesis under the supervision of Professor Matthias Patzold at the University of Agder, Grimstad, Norway. His current research interests include multipath fading channel modeling and channel parameter estimation.

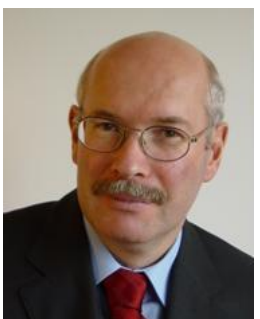

Matthias Pätzold was born in Engelsbach, Germany, in 1958. He received the Dipl.-Ing. and Dr.-Ing. degrees from Ruhr-University Bochum, Bochum, Germany, in 1985 and 1989 respectively, all in Electrical Engineering. In 1998, he received the habil. degree in Communications Engineering from the Technical University of Hamburg-Harburg, Hamburg, Germany. From 1990 to 1992, he was with ANT Nachrichtentechnik GmbH, Backnang, Germany, where he was engaged in digital satellite communications. From 1992 to 2001, he was with the Department of Digital Networks at the Technical University HamburgHarburg. In 2001, he joined the University of Agder, Grimstad, Norway, where he is a full professor for Mobile Communications and the Head of the Mobile Communications Group. He authored and co-authored more than 230 technical journal and conference papers. His publications received nine best paper awards. He is author of the books "Mobile Radio Channels - Modelling, Analysis, and Simulation" (in German) (Wiesbaden, Germany: Vieweg, 1999), "Mobile Fading Channels" (Chichester, U.K.: Wiley \& Sons, 2002), which has been translated into Chinese in 2008, and "Mobile Radio Channels" (Chichester, U.K.: Wiley \& Sons, 2011). Prof. P atzold has been the TPC Chair of ISWCS'07, the TPC Co-Chair of PIMRC'13, ATC'12, ATC'11, ATC'10, ICCE'10, and the Local Organizer of KiVS'01. He has been actively participating in numerous conferences serving as TPC member for more than 20 conferences within the last 3 years. He served as Keynote Speaker, Tutorial Lecturer, and Session Chair for many reputed international conferences. He is an Associate Editor of the IEEE Vehicular Technology Magazine and a member of the International Advisory Board of the Radio Electronics Association of Vietnam (REV). He edited several special issues, including the special issue on "Wireless Future" (Springer, 2009), the special issue on "Trends in Mobile Radio Channels: Modeling, Analysis, and Simulation" (IEEE Vehicular Technology Magazine, 2011), as well as the special issue on 'Modeling and Simulation of Mobile Radio Channels' (Hindawi Publishing Corporation, to appear in 2012). He is a Senior Member of the IEEE. His current research interests include mobile radio communications, especially multipath fading channel modelling, multiple-input multiple-output (MIMO) systems, cooperative communication systems, vehicular-to-vehicular communications, mobile-to-mobile communications, and coded-modulation techniques for fading channels. 